\title{
DESEMPENHO INICIAL DE VARIEDADES DE MELÃO (CUCUMIS MELO L.) SUBMETIDAS A ESTERCO BOVINO
}

\section{INITIAL DEVELOPMENT OF VARIETIES OF MELON (CUCUMIS MELO L) SUBMITTED TO CATTLE MANURE}

\author{
Leandro Alves Pintoㅎ Marcos Silva Tavares ${ }^{2}$; Artur dos Santos Silva ${ }^{3}$ Antonio Alves Pinto ${ }^{4}$; \\ Felipe Thomaz da Camara ${ }^{5}$
}

DOI: https://doi.org/10.31692/978-65-991061-7-0.102-109

\begin{abstract}
RESUMO
A cultura do melão apresenta satisfatória produtividade no Nordeste em virtude da alta exigência em radiação solar, boa adaptabilidade às condições edafoclimáticas, tendo ascensão na demanda e ampliação das áreas de plantio. O Brasil ocupa a décima primeira posição na produtividade mundial, sendo a região Nordeste responsável por $90 \%$ do melão produzido nacionalmente. O objetivo desse trabalho foi avaliar o desempenho germinativo de cinco cultivares de melão: Amarelo, Eldorado, Pele de Sapo, Gália e Japonês. O experimento foi realizado em bandejas de germinação no Centro de Ciências Agrárias e da Biodiversidade CCAB no campus Agronomia em Crato-CE. O substrato usado foi composto por solo/esterco bovino na proporção 1:1. Durante os primeiros dias, colocou-se cobertura morta sobre as bandejas, visando reduzir a variação de temperatura e promover a manutenção da umidade no substrato. A irrigação foi feita diariamente com uso de regador convencional. Os dados foram submetidos à análise de variância (ANAVA) a 5\% de probabilidade e comparados por teste de médias pelo programa estatístico SISVAR, versão 5.3. A cultivar Japonês expressou maior velocidade de emergência e índice de emergência inicial, sendo equivalente às cultivares Amarelo e Eldorado. A estabilização de emergência foi mais rápida avaliando-se a cultivar Japonês e mais lenta na cultivar Pele de sapo. Verifica-se que, em termos de desenvolvimento inicial, o melão japonês, o Amarelo e o Eldorado possuem maior viabilidade. No entanto, a ordem se inverte quando se estuda o consumo; o Amarelo é o mais aceito comercialmente na região. A variedade Gália teve o pior rendimento na porcentagem inicial de emergência.
\end{abstract}

Palavras-Chave: emergência, melão, variedades, seleção.

\begin{abstract}
The melon crop presents satisfactory productivity in the Northeast due to the high demand in solar radiation, good adaptability to the edaphoclimatic conditions, having a rise in demand productivity, being the Northeast region responsible for $90 \%$ of the melon produced nationally. The objective of this work was to evaluate the germinative performance of five melon cultivars: Amarelo, Eldorado, Pele de Sapo, Gaia and Japonês. The experiment was carried out in germination trays at the Center for Agrarian Sciences and Biodiversity - CCAB at the Agronomy campus in Crato-CE. The substrate used was composed of 1: 1 bovine soil / manure. During the first days, mulch was placed on the trays, aiming at reducing temperature variation and promoting the maintenance of moisture in the substrate. The irrigation was done daily with the use of conventional irrigator. Data were submitted to analysis of variance

\footnotetext{
${ }^{1}$ Agronomia, Universidade Federal do Cariri - UFCA, leandroalvespinto96@ gmail.com

${ }^{2}$ Agronomia, Universidade Federal do Cariri - UFCA, marcfilho021@ outlook.com

${ }^{3}$ Agronomia, Universidade Federal do Cariri - UFCA, artur.silva.28@ hotmail.com

${ }^{4}$ Agronomia, Universidade Federal do Cariri - UFCA, antonioufca@ gmail.com

${ }^{5}$ Professor adjunto da Universidade Federal do Cariri - UFCA, felipe.camara@ufca.edu.br
} and expansion of the planting areas. Brazil occupies the eleventh position in the world 
(ANAVA) at $5 \%$ of probability and compared by means of the SISVAR statistical program, version 5.3. The Japanese cultivar showed higher emergence speed and initial emergence index, being equivalent to the cultivars Amarelo and Eldorado. The emergency stabilization was faster by evaluating the Japonês cultivar and slower in the cultivar Pele de sapo. It is found that, in terms of initial development, Japanese melon, Yellow melon and Eldorado have greater viability. However, order is reversed when studying consumption; Yellow is the most commercially accepted in the region. The Galia variety had the worst yield in the initial percentage of emergence.

Keywords: emergence, melon, varieties, selection.

\section{INTRODUÇÃOO}

O melão (Cucumis melo L.) é uma das oleráceas mais populares do mundo, tendo ocupado, em 2013, uma área de 1,18 milhão de hectares, com produção de 29,46 milhões de toneladas, que correspondeu a uma produtividade média de 24,85 t ha-1 (FAO, 2015).

A cultura do melão é uma das que represente maior importância econômica estratégica para a Região Nordeste do Brasil. Esta região detém 95\% da produção nacional. O melão vem tendo grande importância para o comércio de frutas frescas do Brasil. O melão é a principal fruta mais exportada no Brasil, no ano de 2015 foram exportadas $223.746 \mathrm{t}$ de frutos, sendo o Ceará e Rio Grande do Norte os dois maiores estados exportadores de melão do país, responsáveis por $98 \%$ da exportação (SECEX, 2017)

As diferentes condições climáticas existentes no Nordeste brasileiro favorecem o desenvolvimento e produção da cultura do melão com possibilidade de plantios e colheitas durante as diferentes etapas do ano, com limitações apenas nas localidades onde há grande precipitação pluviométrica em determinados períodos (COSTA, 2015).

De acordo (TABIO, 2014), os principais estados produtores de melão em toneladas são: Rio Grande do Norte, Ceará e Bahia, respectivamente. Considerando que em 2012 a produção dos dois maiores estados (Rio Grande do Norte e Ceará) se aproximou em quantidade, com 260.782 toneladas para o Rio Grande do Norte e 219.309 toneladas para Ceará, pois em 2011 a diferença era levemente acentuada com 258.938 toneladas para o primeiro e de 143.466 toneladas para o segundo. Já a terceira maior colocada, Bahia, apresenta valores muito menores que parceiros no ranking, sua produção em quantidade em 2012 é de apenas 34.719 toneladas.

O cultivo de melão no Nordeste apresenta vantagens não só econômicas, mas sustentável e social, levando em consideração que a cultura requer menor quantidade de água para finalização do ciclo e viabiliza a convivência do pequeno produtor no semiárido.

Objetivou-se com esse estudo verificar a potencialidade inicial e desenvolvimento de plântulas das variedades de melão para a região do Cariri Cearense, visando promover 
direcionamento em relação a escolha de materiais com viabilidade para plantio.

\section{FUNDAMENTAÇÃO TEÓRICA}

Os principais produtores de melão no mundo são a China, Turquia, Irã e Egito. O Brasil se destaca na $11^{\circ}$ posição, sendo o $3^{\circ}$ maior produtor da América Latina, com uma produção de 575 mil toneladas, sendo os maiores estados produtores o Rio Grande do Norte e o Ceará. Além de atender o mercado nacional, as exportações brasileiras de melão têm como principal destino Holanda, Reino Unido e Espanha (FAO, 2009; FAO, 2012).

O melão (Cucumis melo L.) é uma cucurbitácea cultivada em várias regiões do mundo e tem grande expressão econômica (DIAS et al., 2014). O cultivo da cultura no Brasil se iniciou na década de 60, onde se estabeleceu em São Paulo e no Rio Grande do Sul, mas devido a sua adaptação as condições edafoclimáticas nas regiões tropicais, no início dos anos 80 propiciou a transferência da cultura para a região do Nordeste brasileiro, onde até os dias atuais predomina a produção da cultura, sendo esta região responsável por $94 \%$ da produção brasileira em 2014 (AGRIANUAL, 2015).

Existe uma grande variedade de melão, com diferentes características, que vão desde a textura e cor da casca, polpa, formato, até o sabor e o tamanho. Os principais tipos de melão são: cantaloupe, amarelo (também conhecido como melão espanhol), charentais, pele de sapo, gália, honey dew e orange flesh. O amarelo e o pele de sapo pertencem ao grupo dos inodoros, enquanto os demais pertencem ao grupo dos aromáticos e são variedades que demandam um manuseio mais cuidadoso e, por isso, devem ser mantidos sob refrigeração. (COMPANHIA DE ENTREPOSTOS..., 2007; GEOCITIES, 2007; GOMES, 2007)

A escolha da cultivar é de fundamental importância para o sucesso da exploração, uma vez que devem ser considerados simultaneamente aspectos de mercado e comercialização, além de qualidades agronômicas quanto à suscetibilidade a doenças e pragas, resistência ao transporte, teor de sólidos solúveis, conservação pós-colheita, entre outras (SILVA et al., 2005; OLIVEIRA et al., 2015).

Dentre os fatores que podem afetar a produção de mudas de boa qualidade, estão incluídos: a qualidade da semente, do substrato e dos insumos utilizado, pois estes contribuem para o desenvolvimento e sanidade da muda (YAMANISHI et al., 2004).

A utilização da matéria orgânica no processo de produção de plântulas é usualmente empregada na melhoria das propriedades físico-químicas do substrato. Ela exerce influência na estrutura, aeração, retenção de umidade e no fornecimento de nutrientes essenciais ao desenvolvimento de culturas (ARAÚJO; PAIVA SOBRINHO, 2011). 


\section{METODOLOGIA}

O presente trabalho foi desenvolvido em casa de vegetação no Centro de Ciências Agrária e da Biodiversidade - CCAB, na Universidade Federal do Cariri- UFCA, Crato-CE, localizada na região do Cariri, com as seguintes coordenadas geográficas: $7^{\circ} 14^{\prime} 3,4^{\prime \prime}$ S e $39^{\circ}$ 22' 7,6" W a 442 m de altitude no período de 06 a 26 de agosto de 2018. De acordo com Lima e Ribeiro (2012), essa região apresenta um clima entre Tropical Semiárido à Tropical Semiárido Brando, com temperatura média entre 24 a $26{ }^{\circ} \mathrm{C}$ e período chuvoso de janeiro a maio possuindo uma média pluviométrica entorno de 925 milímetros de água.

O delineamento experimental utilizado foi o Delineamento Inteiramente Casualidade DIC, com sete repetições e cinco cultivares, totalizando trinta e cinco observações. As variedades estudadas de melão (Cucumis melo L) foram: Amarelo, Eldorado, Pele de Sapo, Gália e japonês todas obtidas de empresa certificada no comércio local.

Os tratamentos foram constituídos por oito células em bandejas de germinação contendo no total 128 células, sendo colocadas duas sementes de melão por célula, com um total 16 sementes por parcela. De acordo com classificação do mapa de solos de média intensidade da FUNCEME (2012), o solo utilizado foi um Argissolo Vermelho Amarelo de relevo suave ondulado e textura da camada superficial do solo classificada como francoarenosa. A constituição química na camada de 0-20 cm foi: pH (1:2,5 H2O): 4,3; P (melich1): 7,0 mg dm-3; K: 0,80 mmolc dm-3; Ca: 2,0 mmolc dm-3; Mg: 3,0 mmolc dm-3; CTC: 26,0 mmolc dm-3 e V (\%): 23. Utilizou-se esterco bovino bem curtido para compor o substrato em uma proporção de $50 \%$ esterco e $50 \%$ de solo.

As irrigações foram efetuadas com irrigador convencional, com turno de rega diária parcelada em duas aplicações (manhã e tarde), objetivando manter o substrato sempre úmido favorecendo assim a melhor germinação e emergência das sementes. Foram analisadas as seguintes variáveis: índice de velocidade de emergência (IVE), o número de dias para estabilizar a emergência (NDE) e a porcentagem de emergência inicial e final (\% EI e \% EF), respectivamente.

O IVE foi determinado conforme metodologia de Maguire (1962), utilizando-se a seguinte fórmula: $I V E=(G 1 / N 1)+(G 2 / N 2)+(G 3 / N 3) \ldots+(G n / N n)$. Onde, G1, G2, G3, ..., Gn são os números de plântulas emergidas após a semeadura e N1, N2, N3, ... Nn é o número de dias que as plântulas levaram para emergir após a semeadura, sendo contado até o último dia de emergência. O NDE foi considerado o último dia que foi observada a emergência de plantulas na parcela, no período de 12 dias (Período de avaliação). A porcentagem de 
emergência inicial foi realizada por meio da contagem das plantas emergidas até o sétimo dia após a semeadura (DAS) e a porcentagem de germinação final, determinada através da avaliação das plântulas emergidas até 12 DAS.

Todos os dados foram submetidos à análise de variância, pelo teste de comparação de médias de Tukey a 5\% de probabilidade, utilizando-se o programa estatístico SISVAR 5,3, versão 5.3.

\section{RESULTADOS E DISCUSSÃO}

Verificar-se que as cultivares obtiveram resultados significativos a $(p>0,01)$, demonstrando comportamentos iniciais diferentes entre si, quando submetidas as mesmas condições de clima (Tabela 1). Essas diferenças podem ser explicadas em função das condições exigidas pelas sementes. Conforme Nogueira et al (2003), a germinação de sementes varia em função do substrato ou de alguma propriedade do mesmo como esterilidade, porosidade, capacidade de retenção de água. As respectivas variedades apresentaram aptidões distintas em relação ao mesmo substrato no qual foram avaliadas, sendo favorecidas ou prejudicadas nos parâmetros estudados: IVE, \%EI, \%EF e NDE.

Tabela1: Síntese da análise de variância, para as variáveis índice de velocidade de Emergência (IVE),

Porcentagem de emergência inicial (\%EI), Porcentagem de emergência final (\%EF) e Número de dias para estabilizar a emergência (NDE).

\begin{tabular}{ccccc}
\hline \multirow{2}{*}{ Fatores } & \multicolumn{4}{c}{ Valores de F } \\
\cline { 2 - 5 } & IVE & \% E & $\% \mathrm{EF}$ & NDE \\
\hline Cultivar & $58,89^{* *}$ & $43,95^{* *}$ & $51,59^{* *}$ & $8,93^{* *}$ \\
\hline CV\% & 15,98 & 19,85 & 13,62 & 14,31 \\
\hline \multicolumn{5}{c}{ Teste de Médias de Tukey $(\mathrm{p}<0,05)$} \\
\hline Cultivares & $6,41 \mathrm{a}$ & $4,16 \mathrm{a}$ & $14,57 \mathrm{ab}$ & $8,57 \mathrm{bc}$ \\
Amarelo & $6,19 \mathrm{ab}$ & $4,02 \mathrm{a}$ & $13,91 \mathrm{ab}$ & $8,00 \mathrm{bc}$ \\
Eldorado & $1,03 \mathrm{c}$ & $0,46 \mathrm{c}$ & $4,37 \mathrm{c}$ & $11,00 \mathrm{c}$ \\
Pele de Sapo & $4,93 \mathrm{~b}$ & $3,02 \mathrm{~b}$ & $12,05 \mathrm{~b}$ & $9,43 \mathrm{ab}$ \\
Gália & $6,52 \mathrm{a}$ & $4,02 \mathrm{a}$ & $15,28 \mathrm{a}$ & $7,28 \mathrm{a}$ \\
Japonês & &
\end{tabular}

Médias seguidas pela mesma letra minúscula na coluna diferem entre si pelo teste de Tukey a $5 \%$ de probabilidade, **: significativo $(\mathrm{P}<0,01)$; *: significativo $(\mathrm{P}<0,05)$; $\mathrm{CV} \%$ : coeficiente de variação.

Observa-se que o coeficiente de variação obtido nas análises foi médio para todos os fatores analisados (Tabela 1), demonstrando que houve um eficiente controle do acaso (Pimentel Gomes, 2009). A análise das cultivares demonstrou que o melão Japonês possuiu uma melhor emergência e maiores medias para os fatores índice de velocidade de emergência, porcentagem de emergência inicial e porcentagem de emergência final. No entanto, não deferiu estatisticamente das cultivares amarelo e eldorado para os mesmos fatores 
mencionados. As cultivares Pele de sapo e Gália tiveram o pior desempenho de emergência inicial para as mesmas, evidenciando um baixo potencial de crescimento primário.

A variedade Pele de sapo pode ser considerada como inviável para o cultivo nas características inerentes ao experimento, sendo a pior em emergência final e diferindo em aproximadamente $300 \%$ das demais.

De acordo com (COSTA, 2010), o melão amarelo é o principal tipo produzido no Nordeste, graças às condições edafoclimáticas, uma vez que suas altas temperaturas e a luminosidade, entre 2000 e 3000 horas no ano da região, juntamente com a umidade relativa, entre $65 \%$ e $75 \%$, propiciam boa produtividade e frutas com elevado teor de açúcares, melhor aroma, sabor e consistência característica.

Entre as características mais estudadas do melão estão o teor de Sólidos Solúveis (SS), que é o fator utilizado para assegurar a qualidade do fruto, estando relacionado à concentração do açúcar no melão, que não deve ser inferior a $10 \%$ (é o chamado BRIX) e a textura ou firmeza da polpa, que indica a resistência para ser transportada e a maior vida nas prateleiras. (SALES JÚNIOR et al., 2004).

A variedade Amarelo relaciona potencial produtivo inicial com facilidade na comercialização. As qualidades de doce em ambas, quando os frutos estão maduros, são praticamente equivalentes: ${ }^{\circ}$ Brix variando de 9-12, na maioria dos casos.

A preferência dos consumidores pode está relacionada à aparência exterior do melão japonês, a qual apresenta estrias peculiares.

Identifica-se que a variável número de dias para estabilizar, a emergência a cultivar japonês não presentou diferença estatística quando comparada com a cultivar Gália, demonstrando que ambas possuem uma rápida estabilização na emergência, as cultivares eldorado, amarelo e pele de sapo apresentaram as piores medias, denotando como consequência uma desigualdade na emergência das plântulas.

\section{CONCLUSÕES}

Verifica-se maiores potenciais de emergência para as variedades Japonês e Amarelo, sendo equivalentes estatisticamente. Contudo, a demanda comercial da região requisita o melão Amarelo como sendo mais vendável.

O melão Pele de sapo pode ser considerado como pouco viável/inviável para cultivo nas condições de experimento as quais foi submetida.

A potencialidade da cultura do melão na região é iminente, uma vez que os fatores climáticos promovem desenvolvimento otimizado quando comparado as demais regiões do 
Brasil.

\section{REFERÊNCIAS}

AGRIANUAL. Anuário da Agricultura Brasileira. São Paulo: FNP Consultoria e Agro Informativos, 2015, 496p.

ARAÚJO, A. P.; PAIVA SOBRINHO, S. Germinação e produção de mudas de tamboril (Enterolobium contortisiliquum (Vell.) Morong) em diferentes substratos. Revista Árvore, Viçosa, v.35, n.3, p. 581-588, maio/jun. 2011.

Companhia de entrepostos e armazéns gerais de são paulo. Disponível em: <http://www.ceagesp.gov.br/>. Acesso em: 8 ago. 2007.

COSTA, N. D. (Ed.). Sistema de produção de melão. Petrolina: Embrapa Semiárido, 2010. (Embrapa Semiárido. Sistemas de Produção, 5)

DIAS, V. G. Crescimento, fisiologia e produção do meloeiro "Pele de sapo" cultivado sob diferentes lâminas de irrigação. 2014. 84 f. Dissertação (Mestrado em Ciências Agrárias) Universidade Estadual da Paraíba - UEPB Campina Grande, 2014.

FAO - Food and Agriculture Organization of the Unites Nations - FAOSTAT - 2009. Faostat database results. Disponível em:<http://faostat.fao.org/faostat/servlet $>$. Acessado em: 29 nov. 2015.

FAO - Food and Agriculture Organization of the Unites Nations - FAOSTAT - 2012. Disponível em: :<wttp://www.fao.org>. Acessado em: 25 set. 2015.

FAO. Agricultural production, primary crops. Disponível em:〈wttp://www.fao.org $>$. Acesso em: 12 dez. 2015.

GEOCITIES. Melão. [S.1.], [2007]. Disponível em: <http://www.geocities.com/> . Acesso em: 5 set. 2007.

GOMES, P. M. Cultivo do melão: manejo, colheita, pós-colheita e comercialização do melão. [S.1]: SENAR, 2007.

OLIVEIRA, J. B. et al. Rendimento e qualidade de frutos de melancia em diferentes épocas de plantio. Revista Caatinga, v. 28, n. 2, p. 19 - 25, 2015.

SALES JÚNIOR, R. et al. Qualidade do melão exportado pelo porto de Natal. Horticultura Brasileira, Brasília, DF, v. 22, n. 1, p. 98-100, 2004.

SECEX- Secretaria de Comércio Exterior. 2015. Disponível em: <http://www.desenvolvimento.gov.br/sitio/interna/interna.php?area=5\&menu=1078\&refr=10 76>. Acesso em 29 de junho de 2017.

SILVA, L. A.; I. R. et al. Estudo de aspectos quantitativos e qualitativos de frutos de genótipos de melão. Revista Ciência Agronômica, v. 36, n. 3, p. 310-315, 2005.

TABIO, Z. F. Estudo da Competitividade da Indústria de Frutas Brasileiras, Melão e 
Manga (2000-2012). TCC (Obtenção de título de Bacharel em Ciências Econômicas com ênfase em Controladoria), Universidade Federal de Alfenas, 24 jul 2014, f.55, Varginha, MG, 24 jul. 2014.

YAMANISHI, O. K.; FAGUNDES, G. R.; MACHADO FILHO, J. A.; VALONE, G. de V. Efeito de diferentes substratos e duas formas de adubação na produção de mudas de mamoeiro. Revista Brasileira de Fruticultura, v. 26, n. 2, p. 276-279, 2004. 